\title{
Downregulation of miR-200c-3p contributes to the resistance of breast cancer cells to paclitaxel by targeting SOX2
}

\author{
JUNQING CHEN $^{1}$, WEI TIAN ${ }^{2}$, HAIFEI HE ${ }^{2}$, FENG CHEN $^{3}$, JIAN HUANG ${ }^{1}$, \\ XIAOJIA WANG $^{1 *}$ and ZHANHONG CHEN ${ }^{1 *}$ \\ ${ }^{1}$ Department of Breast Medical Oncology, Zhejiang Cancer Hospital, Hangzhou, Zhejiang 310022; \\ ${ }^{2}$ Department of Breast Surgical Oncology, The Second Affiliated Hospital, School of Medicine, \\ Zhejiang University, Hangzhou, Zhejiang $310009 ;{ }^{3}$ Department of Breast Surgical Oncology, \\ Zhejiang Cancer Hospital, Hangzhou, Zhejiang 310022, P.R. China
}

Received June 15, 2018; Accepted September 21, 2018

DOI: 10.3892/or.2018.6735

\begin{abstract}
Acquisition of resistance to paclitaxel is a major obstacle to successful treatment of breast cancer patients, but the molecular mechanisms underlying the development of drug resistance remain largely unclear. The aim of the present study was to investigate the role and mechanism of action of miR-200c-3p in the resistance of breast cancer to paclitaxel. It was observed that miR-200c-3p expression, as determined by reverse transcription-quantitative polymerase chain reaction analysis, was significantly downregulated in paclitaxel-resistant MCF-7/Tax cells compared with parental MCF-7 cells. Overexpression of miR-200c-3p increased the chemosensitivity to paclitaxel and enhanced apoptosis in MCF-7/Tax cells, whereas the downregulation of miR-200c-3p exerted the opposite effect. In addition, upregulation of miR-200c-3p in MCF-7/Tax cells suppressed the expression of sex-determining region Y-box 2 (SOX2) at the mRNA and protein levels. Dual-luciferase reporter assay demonstrated that SOX2 is a target of miR-200c-3p in MCF-7/Tax cells. Moreover, knockdown of SOX2 expression increased chemosensitivity to paclitaxel and upregulated miR-200c-3p expression in MCF-7/Tax cells. Taken together, the results of the present study indicated that miR-200c-3p plays a key role in the development of paclitaxel resistance in breast cancer, possibly partially through regulating SOX2 expression, suggesting that
\end{abstract}

Correspondence to: Professor Zhanhong Chen or Professor Xiaojia Wang, Department of Breast Medical Oncology, Zhejiang Cancer Hospital, 38 Guangji Road, Hangzhou, Zhejiang 310022, P.R. China

E-mail: chen11hzhz@sina.com

E-mail: wangxiaojia03@sina.com

*Contributed equally

Key words: miR-200c-3p, breast cancer, chemoresistance, sex-determining region Y-box 2, paclitaxel the miR-200c-3p-SOX2 loop may serve as a potential target for the reversal of paclitaxel resistance in breast cancer

\section{Introduction}

Breast cancer is the most commonly diagnosed malignancy among women and a leading cause of cancer-related mortality worldwide. In the United States, an estimated 266,120 new cases of breast cancer will be diagnosed and 40,920 patients will succumb to this disease in 2018 (1). It has been verified that chemotherapy can improve the survival and quality of life in breast cancer patients (2). Paclitaxel is a chemotherapeutic agent widely used in the treatment of breast cancer. However, $\sim 50 \%$ of breast cancer patients fail to respond to chemotherapeutic agents due to intrinsic or acquired resistance (3). Resistance to chemotherapy remains a major obstacle to successful treatment of breast cancer. Therefore, it is important to investigate the mechanism underlying the development of drug resistance and develop novel therapeutic strategies for overcoming resistance to paclitaxel in breast cancer.

MicroRNAs (miRNAs) are a class of small non-coding RNA molecules that are $19-25$ nucleotides in length and regulate the expression of a wide variety of genes, mainly through degradation of target mRNAs or inhibition of protein translation (4). Previous studies demonstrated that miRNAs are implicated in several critical cellular processes, including proliferation, differentiation, cell-cycle control and apoptosis $(5,6)$. Recently, a growing volume of evidence has demonstrated that dysregulation of miRNAs plays an important role in cancer drug resistance. For example, overexpression of miR-210 was observed to induce caspase-3-mediated apoptosis and reverse gemcitabine resistance by regulation of ABCC5 gene expression in pancreatic cancer (7). It was also observed that loss of miR-17 and miR-20b enhanced breast cancer resistance to paclitaxel through upregulating nuclear receptor coactivator 3 levels (8). Restoration of miR-200c expression inhibited cell proliferation and increased the chemosensitivity to cisplatin by targeting AKT2 in osteosarcoma (9). In female reproductive cancers, class III $\beta$-tubulin was reported to be a target of miR-200c involved in the chemosensitivity to paclitaxel (10). Our previous study demonstrated that downregulation of 
miR-200c was associated with poor response to neoadjuvant chemotherapy in patients with breast cancer (11). However, the molecular mechanisms underlying the role of miR-200c in the resistance of breast cancer to paclitaxel remain largely unexplored.

Sex-determining region Y-box 2 (SOX2) is a member of the SOX gene family that has been demonstrated to play a critical role in the regulation of self-renewal and pluripotency in human embryonic stem cells (12) and activation of breast cancer stem cells (13). Aberrant expression of SOX2 was observed in various types of cancer, such as glioblastoma (14), lung cancer (15) and prostate cancer (16). It was demonstrated that overexpression of SOX2 contributes to resistance of MCF-7 breast cancer cells to tamoxifen, whereas downregulation of SOX2 enhanced the sensitivity of MCF-7 cells to tamoxifen (17). In addition, it was found that knockdown of SOX2 in pancreatic ductal adenocarcinoma cells increased the response to small-molecule inhibitors targeting MEK and AKT signaling (18). A recent study indicated that downregulation of SOX 2 reduced invasiveness and increased sensitivity to paclitaxel by preserving the epithelial-like properties of breast cancer stem cells (19).

The aims of the present study were to determine whether miR-200c-3p expression is significantly downregulated in paclitaxel-resistant breast cancer cells compared with parental cells, to investigate how the overexpression of miR-200c-3p affects the chemosensitivity to paclitaxel and cell apoptosis, and to elucidate the role of SOX 2 in this process.

\section{Materials and methods}

Cell lines. The human breast cancer cell line MCF-7 was obtained from the China Center for Type Culture Collection (Shanghai, China). The paclitaxel-resistant human breast cancer cell line MCF-7/Tax was established through stepwise selection by increasing the concentration of paclitaxel (Sigma-Aldrich; Merck KGaA, Darmstadt, Germany) over 8 months. MCF-7/Tax cells were cultured in the continuous presence of $10 \mu \mathrm{g} / \mathrm{ml}$ paclitaxel to maintain the drug-resistant phenotype (20). Paclitaxel-resistant human breast cancer MCF-7/Tax cells and parental MCF-7 cells were maintained in RPMI-1640 medium (Invitrogen; Thermo Fisher Scientific, Inc., Waltham, MA, USA) supplemented with $10 \%$ fetal bovine serum (Invitrogen; Thermo Fisher Scientific, Inc.) and ampicillin and streptomycin at $37^{\circ} \mathrm{C}$ in a humidified atmosphere containing $5 \% \mathrm{CO}_{2}$. MCF-7/Tax cells were further cultured in drug-free medium for $>2$ weeks prior to subsequent experiments.

miRNA transfection. Cells were seeded in 6-well plates at a density of $10^{5}$ cells per well and cultured for $24 \mathrm{~h}$. The cells were then transfected with miR-200c-3p mimics, miR-200c-3p inhibitor or negative control using Lipofectamine 2000 (Invitrogen; Thermo Fisher Scientific, Inc.) according to the manufacturer's protocol. The miR-200c-3p mimics, miR-200c-3p inhibitor and negative control were purchased from GenePharma Tech (Shanghai, China).

siRNA transfection. Cells were seeded in 6-well plates and transfected with SOX2 siRNA or negative control siRNA using
Lipofectamine 2000 (Invitrogen; Thermo Fisher Scientific, Inc.) according to the manufacturer's protocol. siRNAs were supplied by GenePharma Tech. The sequences used for the siRNAs were as follows: siSOX2, 5'-GGACAUGAUCAGCAU GUAUTT-3' (sense) and 5'-AUACAUGCUGAUCAUGUC CTT-3' (antisense); and negative control siRNA, 5'-UUCUCC GAACGUGUCACGUTT-3' (sense) and 5'-ACGUGACAC GUUCGGAGAATT-3' (antisense). Cells were harvested for further analysis after $48 \mathrm{~h}$.

Cell viability assay. Cell viability was measured using the 3-(4,5-dimethylthiazol-2-yl)-2,5-diphenyltetrazolium bromide (MTT) assay according to the manufacturer's protocol. Untransfected or transfected cells were re-seeded in 96-well plates at a density of $5 \times 10^{3}$ cells per well. The cells were then treated with different concentrations of paclitaxel for 24, 48 and 72 h. A total of $100 \mu \mathrm{l}$ MTT (Sigma-Aldrich; Merck $\mathrm{KGaA}$ ) per well was added and incubated at $37^{\circ} \mathrm{C}$ for $4 \mathrm{~h}$. Subsequently, $150 \mu \mathrm{l}$ dimethyl sulfoxide was added to each well. The absorbance was measured at $570 \mathrm{~nm}$ by a microplate reader (Bio-Rad Laboratories, Inc., Hercules, CA, USA). Three independent experiments were performed in quadruplicate.

Apoptosis assay. Apoptosis was evaluated by flow cytometry using the FITC Annexin V Apoptosis Detection kit I (BD Biosciences, Franklin Lakes, NJ, USA) according to the manufacturer's instructions. The cells were harvested and washed with phosphate-buffered saline. A total of $5 \mu$ l FITC-labeled enhanced Annexin V, $5 \mu \mathrm{l}$ propidium iodide (PI) and $100 \mu \mathrm{l}$ binding buffer were added. After incubation in the dark for $15 \mathrm{~min}$ at room temperature, the samples were analyzed by flow cytometry (FACSCalibur, BD Biosciences). All experiments were performed in triplicate.

Reverse transcription-quantitative polymerase chain reaction $(R T-q P C R)$ analysis. Total RNA from cultured cells was extracted using TRIzol reagent (Invitrogen; Thermo Fisher Scientific, Inc.) according to the manufacturer's protocol. The concentration and quality of the RNA was determined using the Nanodrop 1000 Spectrophotometer (Thermo Fisher Scientific, Inc.). Quantitative analysis of miRNA expression was performed by All-in-One miRNA RT-qPCR Reagent kit (GeneCopoeia, Guangzhou, China) and U6 snRNA was used for normalization according to the manufacturer's instructions. To determine the mRNA levels of SOX2, total RNA was reverse-transcribed using the PrimeScript 1st Strand cDNA Synthesis kit (Takara, Dalian, China) with $\beta$-actin used as an endogenous control according to the manufacturer's instructions. PCR was performed on the StepOnePlus system (ABI 7500; Applied Biosystems; Thermo Fisher Scientific, Inc.) and each sample was run in triplicate. The relative expression levels of miRNA or mRNA were calculated using the comparative $\mathrm{Cq}$ method $\left(2^{-\Delta \Delta \mathrm{Cq}}\right)(21)$. The primers for amplification were as follows: SOX2 forward, 5'-TGGACAGTTACGCGCACAT-3' and reverse, 5'-CGAGTAGGACATGCTGTAGGT-3'; $\beta$-actin forward, 5'-TGACGTGGACATCCGCAAAG-3' and reverse, 5'-CTGGAAGGT GGACAGCGAGG-3'; miR-200c-3p forward, 5'-UAAUACUGCCGGGUAAUGAUGGA-3'; U6 snRNA: 5'-GCTTCGGCAGCACATATACTAAAAT-3'. The 
A

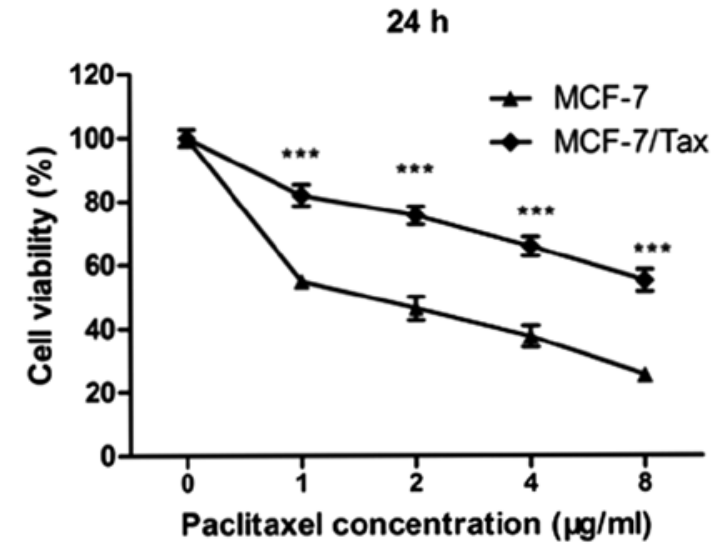

C

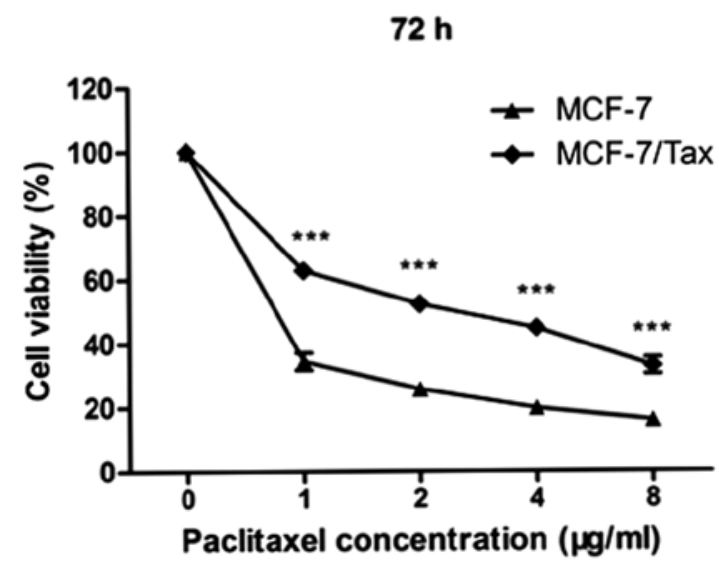

B

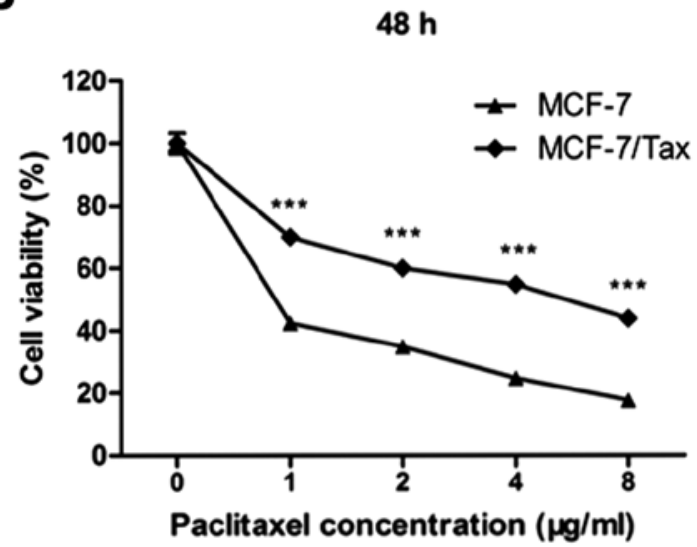

D

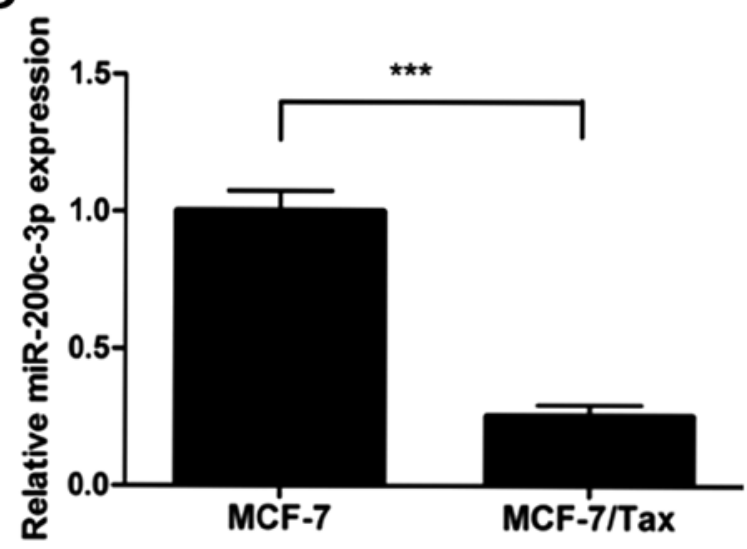

Figure 1. Downregulation of miR-200c-3p in paclitaxel-resistant MCF-7/Tax cells. The viability of MCF-7/Tax cells and parental MCF-7 cells treated with various concentrations of paclitaxel for (A) 24 (B) 48 and (C) $72 \mathrm{~h}$ was measured by MTT assay. The MTT assay demonstrated that MCF-7/Tax cells were significantly resistant to paclitaxel compared with parental MCF-7 cells. (D) miR-200c-3p expression in paclitaxel-resistant MCF-7/Tax cells and parental MCF-7 cells was evaluated using reverse transcription-quantitative polymerase chain reaction analysis. Data are presented as mean \pm standard deviation. ${ }_{* * * * *}^{*}<0.001$.

universal reverse primer of miR-200c-3p and U6 snRNA was 5'-GAGACTGCGGATGTATAGAACTTGA-3'.

Western blotting. Protein extracts were obtained using a lysis buffer and the protein concentration was measured by a bicinchoninic acid protein assay (Thermo Fisher Scientific, Inc.). Total protein was separated on SDS-PAGE gels and transferred to polyvinylidene difluoride membranes (EMD Millipore, Billerica, MA, USA). The membranes were blocked in TBST solution containing 5\% non-fat milk and incubated with the primary antibody for SOX2 (dilution 1:1,000; cat. no. 92494; Abcam, Cambridge, UK) and GAPDH (dilution 1:5,000; cat. no. 201822; Abcam) overnight at $4^{\circ} \mathrm{C}$. GAPDH was used as an internal control. Subsequently, the membranes were incubated with a horseradish peroxidase-conjugated goat anti-rabbit secondary antibody (dilution 1:3,000; cat. no. 6721; Abcam) for $1 \mathrm{~h}$ at room temperature. The bands were visualized using the ECL detection system (Thermo Fisher Scientific, Inc.).

Luciferase reporter assay. The 3'-untranslated region (UTR) of SOX2 containing the miR-200c-3p binding sites and its corresponding mutated sequence were cloned into the psi-CHECK2 vector (Promega Corporation, Madison, WI, USA) downstream of the Renilla luciferase gene. MCF-7/TAX cells were cotransfected with the luciferase vectors and miR-200c-3p mimics or negative control in 96-well plates using Lipofectamine 2000 (Invitrogen; Thermo Fisher Scientific, Inc.). After transfection for $48 \mathrm{~h}$, the Renilla and firefly luciferase activities were measured using the Dual-Luciferase Reporter assay kit (Promega Corp.) according to the manufacturer's protocol. The firefly luciferase activity was normalized to that of Renilla luciferase.

Statistical analysis. The statistical analysis was performed using the SPSS version 19.0 statistical package (IBM Corp., Armonk, NY, USA). The data are presented as mean \pm standard deviation. Differences between two groups were analyzed using the Student's t-test. One way analysis of variance (ANOVA) with Bonferroni post hoc test was used to analyze the differences among three or more groups. $\mathrm{P}<0.05$ was considered to indicate statistically significant differences. 
A

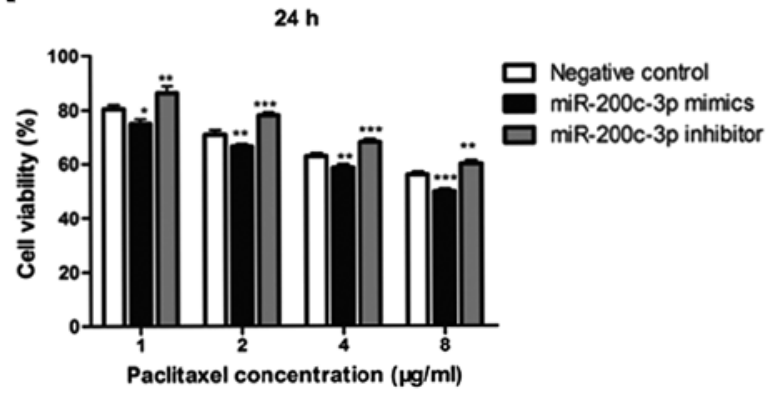

C

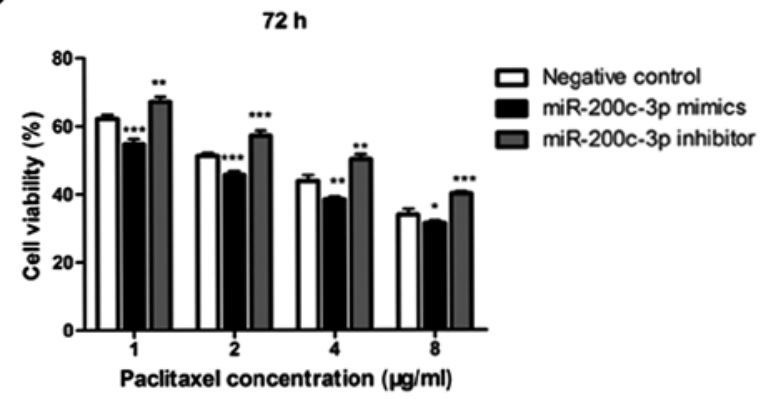

B

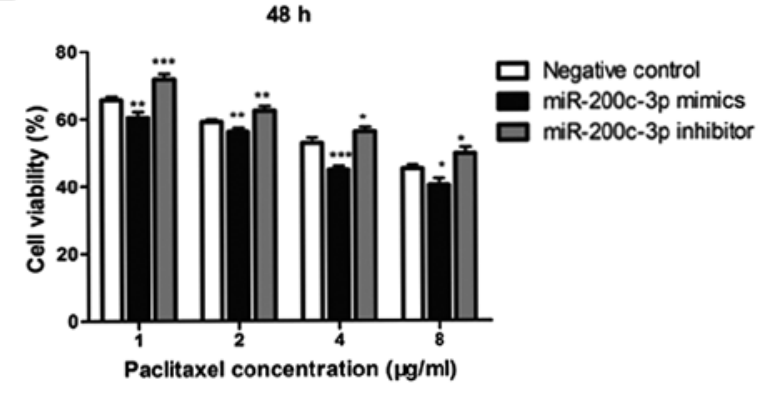

D

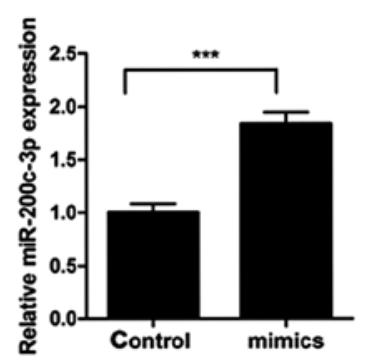

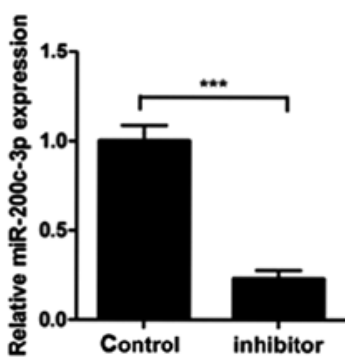

Figure 2. miR-200c-3p overexpression promotes sensitivity of MCF-7/Tax cells to paclitaxel. MCF-7/Tax cells transfected with miR-200c-3p mimics, miR-200c-3p inhibitor or negative control were treated with various concentrations of paclitaxel for (A) 24 (B) 48 and (C) 72 h. Cell viability was measured using the MTT assay. (D) miR-200c-3p expression following transfection of the cells with miR-200c-3p mimics, miR-200c-3p inhibitor or the relative negative control was evaluated using reverse transcription-quantitative polymerase chain reaction analysis. Data are presented as mean \pm standard deviation. ${ }^{*} \mathrm{P}<0.05$, ${ }^{* *} \mathrm{P}<0.01,{ }^{* * *} \mathrm{P}<0.001$.

\section{Results}

miR-200c-3p expression is significantly downregulated in paclitaxel-resistant MCF-7/Tax cells. Paclitaxel-resistant MCF-7/Tax human breast cancer cells were established through stepwise selection by increasing the concentration of paclitaxel in MCF-7 cells. The MCF-7 and MCF-7/Tax cell sensitivity to various concentrations of paclitaxel was determined with MTT assays. As illustrated in Fig. 1A-C, MCF-7/Tax cells were significantly resistant to paclitaxel compared with parental MCF-7 cells (all $\mathrm{P}<0.001$ ). The proliferation of MCF-7/Tax cells was markedly inhibited in a dose- and time-dependent manner.

To determine whether miR-200c-3p expression was implicated in breast cancer cell resistance to paclitaxel, miR-200c-3p expression was evaluated in MCF-7 and MCF-7/Tax cells using RT-qPCR analysis. The expression level of miR-200c-3p in paclitaxel-resistant MCF-7/Tax cells was found to be significantly downregulated by 4 -fold compared with that noted in the parental MCF-7 cells $(\mathrm{P}<0.001$, Fig. 1D). These results suggested that reduced miR-200c-3p expression may be involved in the resistance of breast cancer cells to paclitaxel.

miR-200c-3p overexpression promotes paclitaxel sensitivity in paclitaxel-resistant MCF-7/Tax cells. Downregulation of miR-200c-3p expression was observed in paclitaxel-resistant MCF-7/Tax cells compared with that noted in the parental MCF-7 cells. To evaluate the effect of miR-200c-3p on the resistance to paclitaxel, MCF-7/Tax cells were transfected with miR-200c-3p mimics, miR-200c-3p inhibitor or negative control for $24 \mathrm{~h}$. The cells were then exposed to various concentrations of paclitaxel for 24,48 or $72 \mathrm{~h}$. The cell viability was determined by MTT assays. As shown in Fig. 2A-C, transfection of miR-200c-3p mimics into MCF-7/Tax cells significantly increased the chemosensitivity to paclitaxel. By contrast, downregulation of miR-200c-3p with transfection of miR-200c inhibitors significantly decreased the chemosensitivity to paclitaxel in MCF-7/Tax cells (Fig. 2A-C). miR-200c-3p expression was significantly increased in MCF-7/Tax cells following transfection of miR-200c-3p mimics and significantly decreased in MCF-7/Tax cells transfected with miR-200c-3p inhibitors (Fig. 2D).

miR-200c-3p overexpression promotes apoptosis of paclitaxel-resistant MCF-7/Tax cells. The potential role of miR-200c-3p in the apoptosis of MCF-7/Tax cells was evaluated following transfection of the cells with miR-200c-3p mimics, miR-200c-3p inhibitor or negative control. The cell apoptosis was analyzed using flow cytometry. As shown in Fig. 3A and B, overexpression of miR-200c-3p in MCF-7/Tax cells significantly promoted cell apoptosis compared with the negative control $(\mathrm{P}<0.001)$. By contrast, inhibition of miR-200c-3p in MCF-7/Tax cells significantly decreased apoptosis compared with the negative control $(\mathrm{P}<0.01)$. In addition, a marked increase in the apoptotic rate was observed in the MCF-7/Tax cells transfected with miR-200c-3p mimics following treatment with $5 \mu \mathrm{g} / \mathrm{ml}$ paclitaxel for $48 \mathrm{~h}$ compared with the negative control, whereas a significant decrease in the apoptotic rate was detected in MCF-7/Tax cells transfected with miR-200c-3p inhibitor following paclitaxel treatment 
A
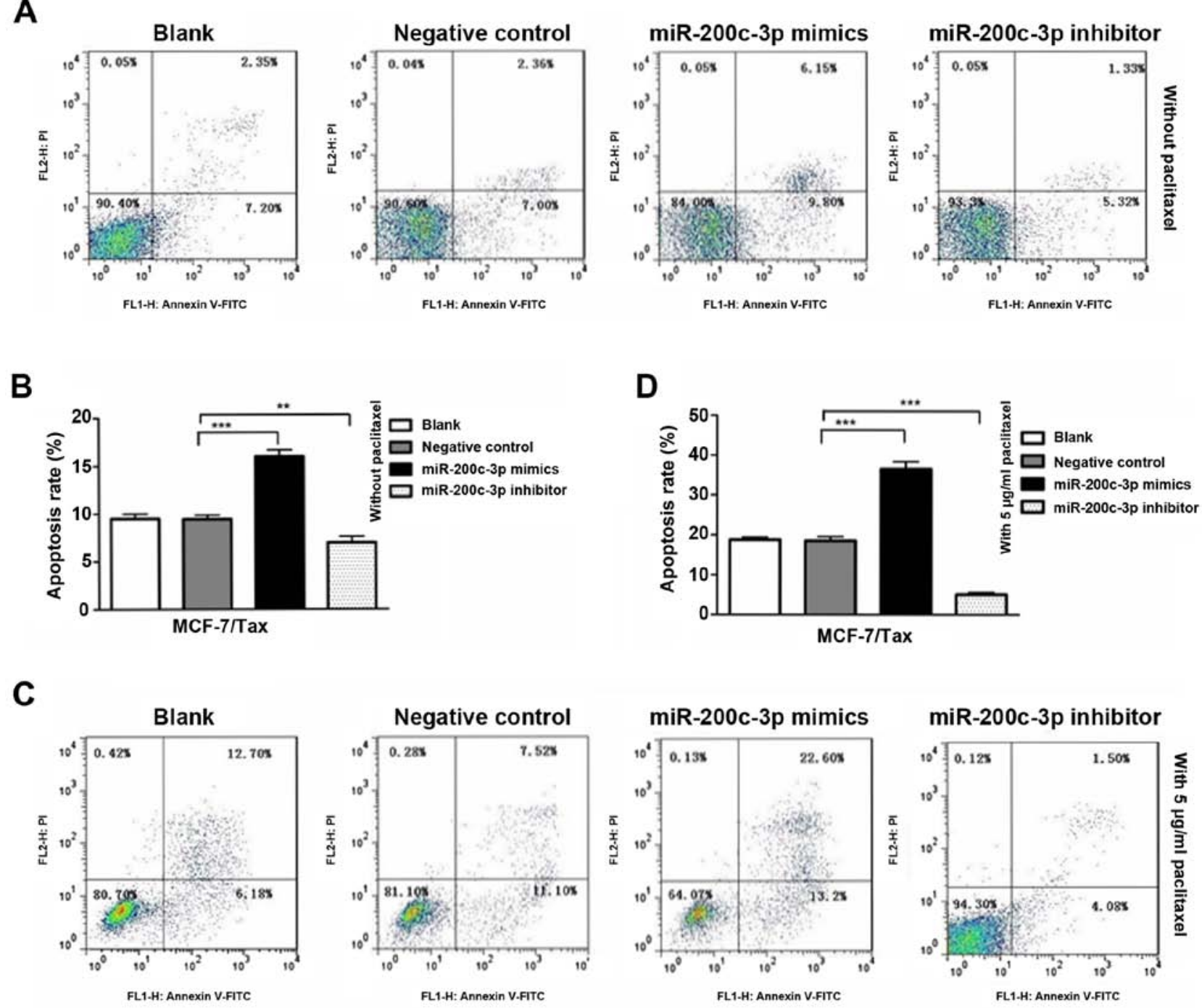

Figure 3. miR-200c-3p overexpression promotes apoptosis of MCF-7/Tax cells with or without paclitaxel treatment. The cell apoptosis rate was analyzed by flow cytometry. Apoptosis rate of MCF-7/Tax cells transfected with miR-200c-3p mimics, miR-200c-3p inhibitor or negative control (A and B) without paclitaxel and (C and D) with $5 \mu \mathrm{g} / \mathrm{ml}$ paclitaxel for $48 \mathrm{~h}$. Data are presented as mean \pm standard deviation. ${ }^{* *} \mathrm{P}<0.01,{ }^{* * *} \mathrm{P}<0.001$.

compared with the negative control $(\mathrm{P}<0.001$, Fig. $3 \mathrm{C}$ and $\mathrm{D})$. These findings indicate that overexpression of miR-200c-3p promoted MCF-7/Tax cell apoptosis, with or without paclitaxel treatment, whereas the downregulation of miR-200c-3p exerted the opposite effect.

Prediction and validation of SOX2 as a target of $\mathrm{miR}-200 c-3 p$. To further explore the molecular mechanism of action of miR-200c-3p in the resistance of breast cancer cells to paclitaxel, target gene prediction of miR-200c-3p was performed using the TargetScan database, one of the most widely used miRNA target prediction algorithms. Bioinformatic analysis indicated that the SOX2 gene included the putative miR-200c-3p targeting sequence. The predicted interaction between miR-200c-3p and its target site in the SOX2 3' UTR is illustrated in Fig. 4A.

The expression of SOX2 at the mRNA level was evaluated in MCF-7/Tax and MCF-7 cells using RT-qPCR analysis. The results demonstrated that SOX2 mRNA expression in MCF-7/Tax cells was significantly increased by 2 -fold compared with parental MCF-7 cells ( $\mathrm{P}<0.01$, Fig. 4B). Consistent with SOX2 mRNA expression, the SOX2 protein expression in MCF-7/Tax cells, as determined by western blotting, was also increased compared with parental MCF-7 cells (Fig. 4C).

To determine whether SOX2 is a direct target of miR-200c-3p, a dual luciferase reporter assay was performed. MCF-7/Tax cells were co-transfected with the SOX2 reporter or empty vector and miR-200c-3p mimic or miR-negative control. At $48 \mathrm{~h}$ after transfection, the Renilla and firefly luciferase activities were measured using the Dual-Luciferase Reporter assay kit. As shown in Fig. 4D, the luciferase activity was markedly suppressed in the presence of miR-200c-3p mimics, whereas no reduction in luciferase activity was observed with the empty vector control or in the presence of miR-negative control.

Upregulation of miR-200c-3p in MCF-7/Tax cells suppresses SOX 2 expression. To further verify the effect of miR-200c-3p on SOX2 gene expression in paclitaxel-resistant breast 


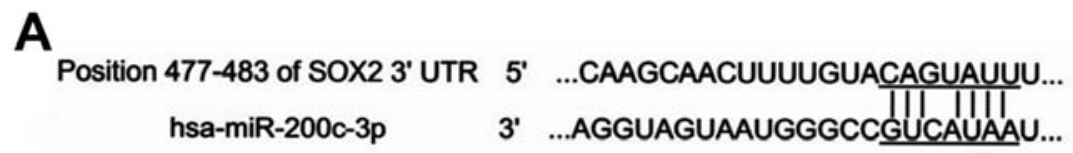

C

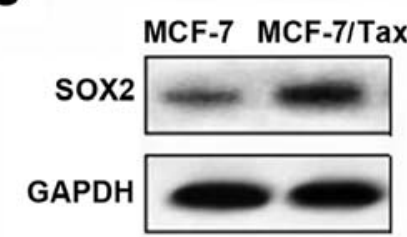

B

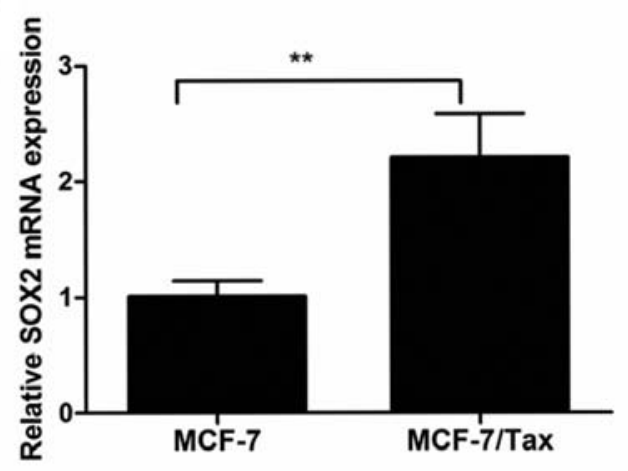

D

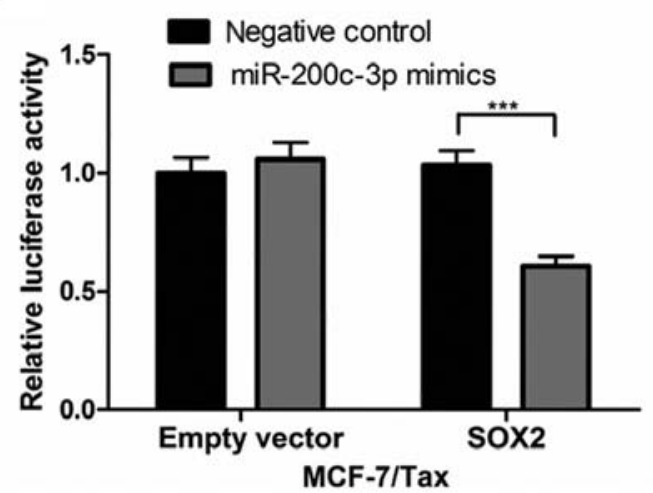

Figure 4. SOX2 is a target of miR-200c-3p. (A) Putative binding sites of miR-200c-3p in the SOX2 3' UTR as predicted by TargetScan. (B) Increased SOX2 mRNA expression, as determined by reverse transcription-quantitative polymerase chain reaction analysis, was observed in MCF-7/Tax cells compared with MCF-7 cells. (C) Increased SOX2 protein expression determined by western blotting in MCF-7/Tax cells compared with MCF-7 cells. (D) Luciferase activity in MCF-7/Tax cells was measured by the dual luciferase reporter assay. Data are presented as mean \pm standard deviation. ${ }^{* * *} \mathrm{P}<0.01,{ }^{* * * *} \mathrm{P}<0.001 . \mathrm{SOX} 2$, sex-determining region Y-box 2.
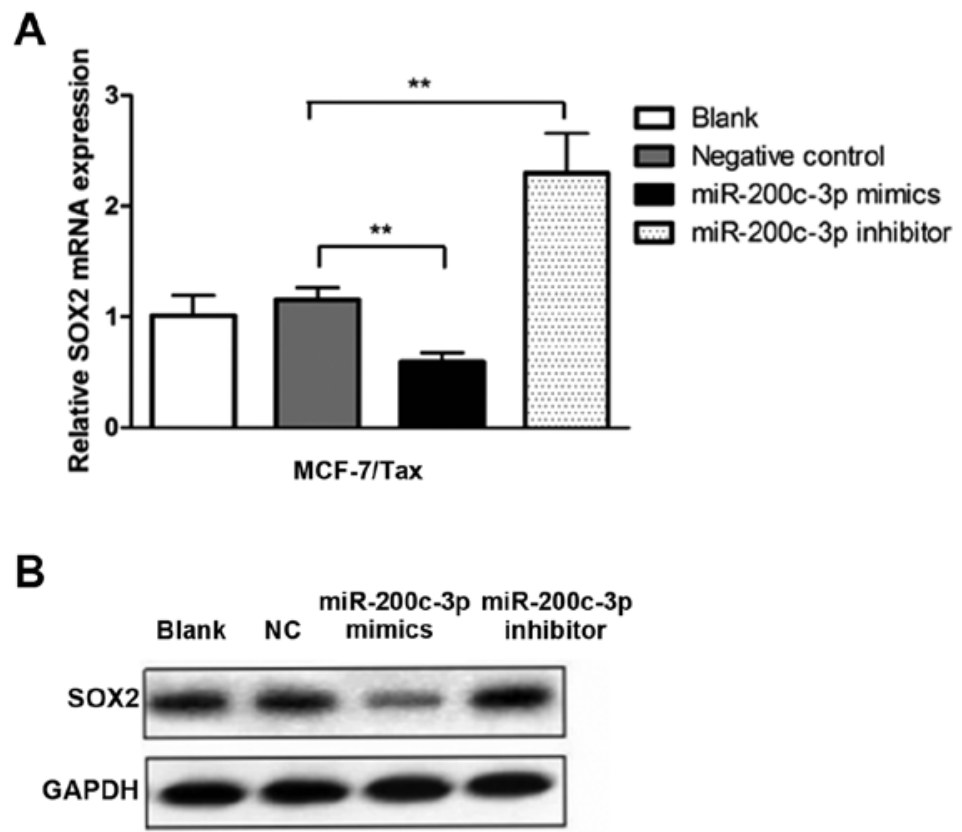

Figure 5. miR-200c-3p overexpression inhibits SOX2 expression. (A) Reverse transcription-quantitative polymerase chain reaction analysis of SOX2 mRNA in MCF-7/Tax cells transfected with miR-200c-3p mimics, miR-200c-3p inhibitor or negative control. (B) Western blot analysis of SOX2 protein expression in MCF-7/Tax cells transfected with miR-200c-3p mimics, miR-200c-3p inhibitor or negative control. Data are presented as mean \pm standard deviation. ${ }^{* *} \mathrm{P}<0.01$. SOX2, sex-determining region Y-box 2.

cancer cells, the SOX2 mRNA and protein expression in MCF-7/Tax cells was determined following transfection with miR-200c-3p mimics, miR-200c-3p inhibitor or negative control. It was observed that miR-200c-3p overexpression in the MCF-7/Tax cells resulted in a significant decrease in SOX2 mRNA compared with the negative control $(\mathrm{P}<0.01$, Fig. $5 \mathrm{~A})$. By contrast, downregulation of miR-200c-3p significantly increased the expression of SOX 2 mRNA in the MCF-7/Tax 

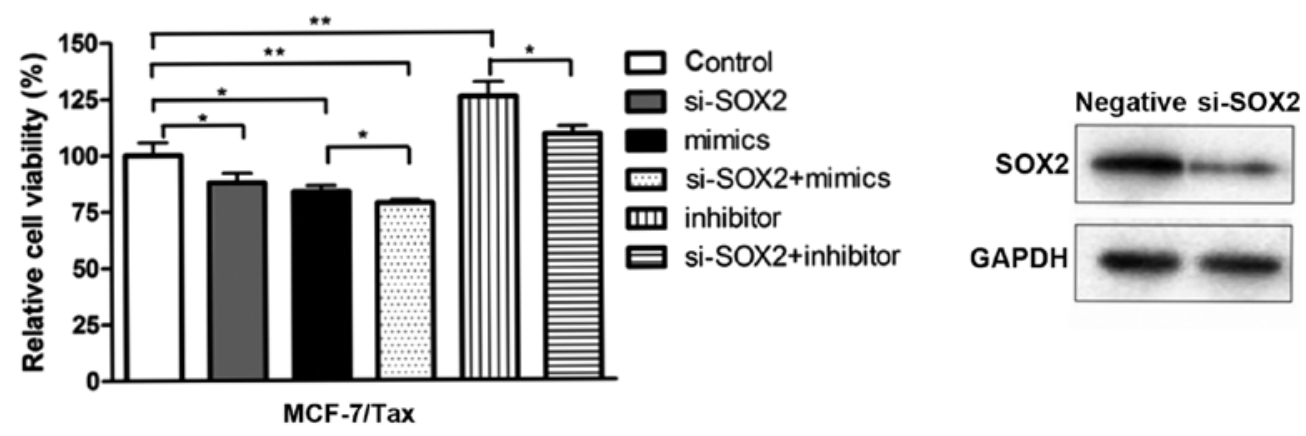

Figure 6. SOX2 knockdown increases chemosensitivity to paclitaxel in MCF-7/Tax cells. MCF-7/Tax cells were transfected with siRNA-SOX2, miR-200c-3p mimics, miR-200c-3p inhibitor, siRNA-SOX2 combined with miR-200c-3p mimics, siRNA-SOX2 combined with miR-200c-3p inhibitor or negative control. Cells were exposed to $5 \mu \mathrm{g} / \mathrm{ml}$ paclitaxel for $48 \mathrm{~h}$ and cell viability was determined by using MTT assays (left histogram). The transfection efficiency was detected by western blot analysis (right blot). Data are presented as mean \pm standard deviation. ${ }^{*} \mathrm{P}<0.05,{ }^{* *} \mathrm{P}<0.01$. SOX2, sex-determining region $\mathrm{Y}$-box 2 .

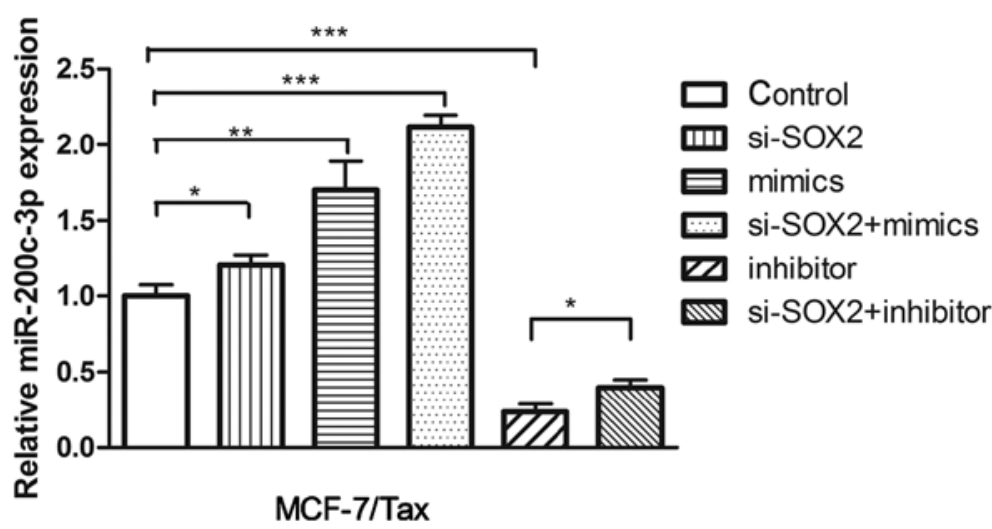

Figure 7. SOX2 knockdown upregulates miR-200c-3p expression in MCF-7/Tax cells. miR-200c-3p expression was evaluated by using reverse transcription-quantitative polymerase chain reaction analysis. Data are presented as mean \pm standard deviation. ${ }^{*} \mathrm{P}<0.05,{ }^{* * *} \mathrm{P}<0.01,{ }^{* * * *} \mathrm{P}<0.001$. SOX2, sex-determining region Y-box 2.

cells compared with the negative control $(\mathrm{P}<0.01$, Fig. 5A). As shown in Fig. 5B, overexpression of miR-200c-3p in MCF-7/Tax cells also suppressed the expression of the SOX2 protein, whereas downregulation of miR-200c-3p exerted the opposite effect.

SOX2 knockdown increases chemosensitivity to paclitaxel in MCF-7/Tax cells. To investigate whether SOX2 is implicated in the resistance of breast cancer cells to paclitaxel, the paclitaxel-resistant MCF-7/Tax cells were transfected with siRNA-SOX2, miR-200c-3p mimics, miR-200c-3p inhibitor, siRNA-SOX2 combined with miR-200c-3p mimics, siRNA-SOX2 combined with miR-200c-3p inhibitor or negative control. Subsequently, the transfected cells were exposed to $5 \mu \mathrm{g} / \mathrm{ml}$ paclitaxel for $48 \mathrm{~h}$ and cell viability was determined with MTT assays. The transfection efficiency of MCF-7/Tax cells transfected with siRNA-SOX2 was detected by western blot analysis (Fig. 6). As shown in Fig. 6, transfection with miR-200c-3p mimics and siRNA-SOX2 reduced cell viability more prominently compared with transfection with miR-200c-3p mimics alone $(\mathrm{P}<0.05)$. In addition, treatment with miR-200c-3p inhibitor and siRNA-SOX2 also reduced cell viability compared with treatment with miR-200c-3p inhibitor alone $(\mathrm{P}<0.05)$. It was observed that SOX2 knockdown was able to increase the sensitivity of MCF-7/Tax cells to paclitaxel compared with the control.
SOX2 knockdown in MCF-7/Tax cells upregulates miR-200c-3p expression. To investigate the potential effect of SOX 2 on miR-200c-3p expression, siRNA interference was used to knock down SOX2 in MCF-7/Tax cells. The expression level of miR-200c-3p was determined by RT-qPCR. As shown in Fig. 7, SOX2 knockdown resulted in significantly increased levels of miR-200c-3p expression in MCF-7/Tax cells compared with the control $(\mathrm{P}<0.05)$. In addition, co-treatment with miR-200c-3p mimics and siRNA-SOX2 significantly increased miR-200c-3p expression in MCF-7/Tax cells $(\mathrm{P}<0.001$, Fig. 7). Treatment with siRNA-SOX2 and miR-200c-3p inhibitor significantly upregulated miR-200c-3p expression in MCF-7/Tax cells compared following treatment with the miR-200c inhibitor alone $(\mathrm{P}<0.05$, Fig. 7$)$.

\section{Discussion}

Although notable improvements have been made in the treatment of breast cancer over the past decades, drug resistance remains a major obstacle to successful treatment of breast cancer patients. Recent studies have demonstrated that aberrant miR-200c expression may play an important role in cancer chemotherapeutic resistance. Decreased miR-200c expression was previously observed in cisplatin-resistant breast cancer cells compared to parental MCF-7 breast cancer cells (22). In addition, Zhu et al reported that miR-200c expression was 
downregulated in vincristine-resistant gastric cancer cells compared with parental SGC7901 gastric cancer cells (23). miR-200c expression was also downregulated in cisplatin-resistant lung cancer cells compared with parental A549 lung cancer cells (23). In our previous study, we demonstrated that the miR-200c expression level in doxorubicin-resistant breast cancer cells was markedly downregulated compared with that in MCF-7 breast cancer cells (11). In the present study, miR-200c-3p expression in paclitaxel-resistant breast cancer cells was found to be significantly downregulated compared with parental MCF-7 breast cancer cells. In addition, overexpression of miR-200c-3p in MCF-7/Tax cells enhanced the chemosensitivity to paclitaxel, while downregulation of miR-200c-3p decreased the chemosensitivity to paclitaxel in MCF-7/Tax cells. Furthermore, it was demonstrated that overexpression of miR-200c-3p promoted MCF-7/Tax cell apoptosis, with or without paclitaxel treatment. These findings indicate that downregulation of miR-200c-3p expression is involved in the resistance of breast cancer cells to paclitaxel, which was consistent with previous reports. However, Hamano et al reported that miR-200c expression was increased in cisplatin-resistant esophageal cancer cells and overexpression of miR-200c induced chemoresistance in esophageal cancer (24). The inconsistent correlation between miR-200c expression and chemoresistance may be due, in part, to the differences among tumor types.

SOX2 has been demonstrated to play an important role in the maintenance of breast cancer stem cells (13). A previous study indicated that SOX2 may be implicated in drug resistance, and downregulation of SOX2 may enhance chemosensitivity of breast cancer cells to paclitaxel (19). Overexpression of SOX2 was demonstrated to enhance the resistance of PC-3 prostate cancer cells to paclitaxel by promoting cell proliferation and preventing cell apoptosis (16). The miR-200 family was found to be downregulated in human breast cancer stem cells as well as mammary stem cells, suggesting an association between the miR-200 family and stemness (25). In the present study, SOX2 mRNA and SOX2 protein expression in paclitaxel-resistant MCF-7/Tax cells were found to be markedly increased compared with their parental MCF-7 cells, while miR-200c-3p expression in MCF-7/Tax cells was downregulated compared with MCF-7 cells. Moreover, upregulation of miR-200c-3p in MCF-7/Tax cells suppressed the expression of SOX2 at the mRNA and protein levels, whereas the downregulation of miR-200c-3p exerted the opposite effect. The luciferase reporter assay revealed that SOX 2 was a direct target of miR-200c-3p in breast cancer. It was demonstrated that a single miRNA could regulate tens or hundreds of target genes, and one gene could also be regulated by multiple miRNAs. Antiapoptotic factors, such as the B-cell lymphoma-2 (BCL-2) and X-linked inhibitor of apoptosis protein (XIAP) genes, were found to be upregulated in vincristine-resistant gastric cancer cells and cisplatin-resistant lung cancer cells (23). BCL-2 and XIAP were identified as target genes of miR-200c and were involved in drug resistance (23). In clear-cell renal cell carcinoma, miR-200c was reported to enhance the efficiency of sorafenib and imatinib by targeting heme oxygenase-1 (HO-1) (26). The findings of the study suggest that downregulation of miR-200c-3p contributes to paclitaxel resistance in breast cancer cells, at least partially through targeting the SOX2 gene.
It was demonstrated that overexpression of the miR-200 family upregulated E-cadherin expression, whereas inhibition of the miR-200 family reduced E-cadherin expression $(27,28)$. The miR-200 family was demonstrated to play a key role in epithelial-to-mesenchymal transition (EMT) by targeting the E-cadherin transcriptional repressors ZEB1 and ZEB2 (29,30). Interestingly, ZEB1 and ZEB2 were also found to repress miR-200 family transcription by binding to their regulatory E-boxes $(29,30)$. Therefore, ZEB1/ZEB2 and the miR-200 family formed a double-negative feedback loop involved in tumor progression and metastasis $(29,30)$. In the present study, the knockdown of SOX2 using SOX2 siRNA markedly increased miR-200c-3p expression in paclitaxel-resistant MCF-7/Tax cells. Furthermore, knockdown of SOX2 in MCF-7/Tax cells increased chemosensitivity to paclitaxel. A previous study reported that miR-200 expression was regulated by SOX2 through contributing to the cell cycle exit and neuronal differentiation of neural stem/progenitor cells (31). It was indicated that the transcription of the mmu-miR-200c/141 gene cluster was activated by SOX2 through binding to their promoters (31). In colorectal carcinoma, it was demonstrated that the miR-200c-SOX2 negative feedback loop mechanism was involved in the regulation of cell stemness, proliferation and metastasis (32). It was found that SOX2 could bind to specific promoter transcription factor binding sites of miR-200c and inhibit the transcription (32).

In summary, we herein demonstrated that miR-200c-3p plays a crucial role in the resistance of breast cancer cells to paclitaxel, possibly partially through targeting SOX2. Furthermore, the evidence provided supports the role of SOX2 in the regulation of miR-200c-3p expression. Taken together, the findings of the present study point to the miR-200c-3p/SOX2 loop as a promising future therapeutic target for overcoming paclitaxel resistance in breast cancer patients.

\section{Acknowledgements}

Not applicable.

\section{Funding}

The present study was supported by grants from the Natural Science Foundation of Zhejiang Province (LQ13H160016) and the Medical Science and Technology Program of Zhejiang Province (2013KYA026 and 2015DTA004) and the National Nature Science Foundation of China (81672597).

\section{Availability of data and materials}

All the datasets generated/analyzed in the present study are available from the corresponding author on reasonable request.

\section{Authors' contributions}

JC, XW and ZC conceived and designed the study. JC, WT, $\mathrm{HH}, \mathrm{FC}$ and $\mathrm{JH}$ performed the experiments. JC and WT wrote the manuscript. $\mathrm{HH}, \mathrm{FC}, \mathrm{JH}, \mathrm{XW}$ and $\mathrm{ZC}$ reviewed and edited the manuscript. All authors read and approved the manuscript and agree to be accountable for all aspects of the research in 
ensuring that the accuracy or integrity of any part of the work are appropriately investigated and resolved.

\section{Ethics approval and consent to participate}

Not applicable.

\section{Patient consent for publication}

Not applicable.

\section{Competing interests}

The authors declare that they have no competing interests to disclose.

\section{References}

1. Siegel RL, Miller KD and Jemal A: Cancer statistics, 2018. CA Cancer J Clin 68: 7-30, 2018.

2. Hassan MS, Ansari J, Spooner D and Hussain SA: Chemotherapy for breast cancer (Review). Oncol Rep 24: 1121-1131, 2010.

3. O'Driscoll L and Clynes M: Biomarkers and multiple drug resistance in breast cancer. Curr Cancer Drug Targets 6: 365-384, 2006.

4. Ng EK, Wong CL, Ma ES and Kwong A: MicroRNAs as new players for diagnosis, prognosis, and therapeutic targets in breast cancer. J Oncol 2009: 305420, 2009.

5. Bartel DP: MicroRNAs: Genomics, biogenesis, mechanism, and function. Cell 116: 281-297, 2004.

6. Lim LP, Lau NC, Garrett-Engele P, Grimson A, Schelter JM, Castle J, Bartel DP, Linsley PS and Johnson JM: Microarray analysis shows that some microRNAs downregulate large numbers of target mRNAs. Nature 433: 769-773, 2005.

7. Amponsah PS, Fan P, Bauer N, Zhao Z, Gladkich J, Fellenberg J and Herr I: microRNA-210 overexpression inhibits tumor growth and potentially reverses gemcitabine resistance in pancreatic cancer. Cancer Lett 388: 107-117, 2017.

8. Ao X, Nie P, Wu B, Xu W, Zhang T, Wang S, Chang H and Zou Z: Decreased expression of microRNA-17 and microRNA-20b promotes breast cancer resistance to taxol therapy by upregulation of NCOA3. Cell Death Dis 7: e2463, 2016.

9. Liu Y, Zhu ST, Wang X, Deng J, Li WH, Zhang P and Liu BS: MiR-200c regulates tumor growth and chemosensitivity to cisplatin in osteosarcoma by targeting AKT2. Sci Rep 7: 13598, 2017.

10. Cochrane DR, Howe EN, Spoelstra NS and Richer JK: Loss of miR-200c: A marker of aggressiveness and chemoresistance in female reproductive cancers. J Oncol 2010: 821717, 2010.

11. Chen J, Tian W, Cai H, He H and Deng Y: Down-regulation of microRNA-200c is associated with drug resistance in human breast cancer. Med Oncol 29: 2527-2534, 2012

12. Fong H, Hohenstein KA and Donovan PJ: Regulation of self-renewal and pluripotency by Sox 2 in human embryonic stem cells. Stem Cells 26: 1931-1938, 2008.

13. Leis O, Eguiara A, Lopez-Arribillaga E, Alberdi MJ, Hernandez-Garcia S, Elorriaga K, Pandiella A, Rezola R and Martin AG: Sox 2 expression in breast tumours and activation in breast cancer stem cells. Oncogene 31: 1354-1365, 2012.

14. Alonso MM, Diez-Valle R, Manterola L, Rubio A, Liu D, Cortes-Santiago N, Urquiza L, Jauregi P, Lopez de Munain A, Sampron N, et al: Genetic and epigenetic modifications of Sox2 contribute to the invasive phenotype of malignant gliomas. PLoS One 6: e26740, 2011.

15. Rudin CM, Durinck S, Stawiski EW, Poirier JT, Modrusan Z, Shames DS, Bergbower EA, Guan Y, Shin J, Guillory J, et al: Comprehensive genomic analysis identifies SOX2 as a frequently amplified gene in small-cell lung cancer. Nat Genet 44: 1111-1116, 2012.
16. Li D, Zhao LN, Zheng XL, Lin P, Lin F, Li Y, Zou HF, Cui RJ, Chen $\mathrm{H}$ and Yu XG: Sox 2 is involved in paclitaxel resistance of the prostate cancer cell line PC-3 via the PI3K/Akt pathway. Mol Med Rep 10: 3169-3176, 2014.

17. Piva M, Domenici G, Iriondo O, Rábano M, Simões BM, Comaills V, Barredo I, López-Ruiz JA, Zabalza I, Kypta R and Vivanco MD: Sox 2 promotes tamoxifen resistance in breast cancer cells. EMBO Mol Med 6: 66-79, 2014.

18. Wuebben EL, Wilder PJ, Cox JL, Grunkemeyer JA, Caffrey T, Hollingsworth MA and Rizzino A: SOX2 functions as a molecular rheostat to control the growth, tumorigenicity and drug responses of pancreatic ductal adenocarcinoma cells. Oncotarget 7: 34890-34906, 2016.

19. Mukherjee P, Gupta A, Chattopadhyay D and Chatterji U: Modulation of SOX2 expression delineates an end-point for paclitaxel-effectiveness in breast cancer stem cells. Sci Rep 7: 9170, 2017.

20. Yang Q, Wang Y, Lu X, Zhao Z, Zhu L, Chen S, Wu Q, Chen C and Wang Z: MiR-125b regulates epithelial-mesenchymal transition via targeting Sema4C in paclitaxel-resistant breast cancer cells. Oncotarget 6: 3268-3279, 2015.

21. Livak KJ and Schmittgen TD: Analysis of relative gene expression data using real-time quantitative PCR and the 2(-Delta Delta C(T)) method. Methods 25: 402-408, 2001.

22. Pogribny IP, Filkowski JN, Tryndyak VP, Golubov A, Shpyleva SI and Kovalchuk O: Alterations of microRNAs and their targets are associated with acquired resistance of MCF-7 breast cancer cells to cisplatin. Int J Cancer 127: 1785-1794, 2010.

23. Zhu W, Xu H, Zhu D, Zhi H, Wang T, Wang J, Jiang B, Shu Y and Liu P: miR-200bc/429 cluster modulates multidrug resistance of human cancer cell lines by targeting BCL2 and XIAP. Cancer Chemother Pharmacol 69: 723-731, 2012.

24. Hamano R, Miyata H, Yamasaki M, Kurokawa Y, Hara J Moon JH, Nakajima K, Takiguchi S, Fujiwara Y, Mori M and Doki Y: Overexpression of miR-200c induces chemoresistance in esophageal cancers mediated through activation of the Akt signaling pathway. Clin Cancer Res 17: 3029-3038, 2011.

25. Shimono Y, Zabala M, Cho RW, Lobo N, Dalerba P, Qian D, Diehn M, Liu H, Panula SP, Chiao E, et al: Downregulation of miRNA-200c links breast cancer stem cells with normal stem cells. Cell 138: 592-603, 2009.

26. Gao C, Peng FH and Peng LK: MiR-200c sensitizes clear-cell renal cell carcinoma cells to sorafenib and imatinib by targeting heme oxygenase-1. Neoplasma 61: 680-689, 2014.

27. Park SM, Gaur AB, Lengyel E and Peter ME: The miR-200 family determines the epithelial phenotype of cancer cells by targeting the E-cadherin repressors ZEB1 and ZEB2. Genes Dev 22: 894-907, 2008.

28. Korpal M, Lee ES, Hu G and Kang Y: The miR-200 family inhibits epithelial-mesenchymal transition and cancer cell migration by direct targeting of E-cadherin transcriptional repressors ZEB1 and ZEB2. J Biol Chem 283: 14910-14914, 2008.

29. Brabletz S and Brabletz T: The ZEB/miR-200 feedback loop-a motor of cellular plasticity in development and cancer. EMBO Rep 11: 670-677, 2010.

30. Hill L, Browne G and Tulchinsky E: ZEB/miR-200 feedback loop: At the crossroads of signal transduction in cancer. Int $\mathrm{J}$ Cancer 132: 745-754, 2013.

31. Peng C, Li N, Ng YK, Zhang J, Meier F, Theis FJ, Merkenschlager M, Chen W, Wurst W and Prakash N: A unilateral negative feedback loop between miR-200 microRNAs and Sox $2 / E 2 F 3$ controls neural progenitor cell-cycle exit and differentiation. J Neurosci 32: 13292-13308, 2012.

32. Lu YX, Yuan L, Xue XL, Zhou M, Liu Y, Zhang C, Li JP, Zheng L, Hong M and Li XN: Regulation of colorectal carcinoma stemness, growth, and metastasis by an miR-200c-Sox 2-negative feedback loop mechanism. Clin Cancer Res 20: 2631-2642, 2014. 